CORIGINAL ARTICLE

Volume 16 Issue 22021

DOI: 10.21315/aos2021.16.2.3

ARTICLE INFO

Submitted: $25 / 9 / 2020$

Accepted: $17 / 6 / 2021$

Online: 22/12/2021

\section{Sensitivity and Specificity of Different Diagnostic Methods in Occlusal Caries Detection of Permanent Teeth Among Paediatric Patients}

\author{
Ilham Wan Mokhtara, ${ }^{a,{ }^{*}}$, Annapurny Venkiteswaran ${ }^{\mathrm{b}}$, Mohd Yusmiaidil
} Putera Mohd Yusof ${ }^{c, d}$

${ }^{a}$ Centre for Comprehensive Care Studies, Faculty of Dentistry, Universiti Teknologi MARA, 47000 Sungai Buloh, Selangor, Malaysia

${ }^{b}$ Centre for Pediatric Dentistry and Orthodontics Studies, Faculty of

Dentistry, Universiti Teknologi MARA, 47000 Sungai Buloh, Selangor, Malaysia

${ }^{c}$ Centre for Oral and Maxillofacial Diagnostic and Medicine Studies, Faculty of Dentistry, Universiti Teknologi MARA, 47000 Sungai Buloh, Selangor, Malaysia

${ }^{d}$ Institute of Pathology, Laboratory and Forensic Medicine (I-PPerForM), Universiti Teknologi MARA, 47000 Sungai Buloh, Selangor, Malaysia

*Corresponding author: ilham@uitm.edu.my

To cite this article: Mokhtar IW, Venkiteswaran A, Yusof MYPM (2021). Sensitivity and specificity of different diagnostic methods in occlusal caries detection of permanent teeth among paediatric patients. Arch Orofac Sci, 16(2): 113-126. https://doi.org/10.21315/aos2021.16.2.3

To link to this article: https://doi.org/10.21315/aos2021.16.2.3

\title{
ABSTRACT
}

Dental caries is a commonly progressive disease that proceeds through various degrees of severity that a dentist can detect. The aims of the in vivo study were to assess the accuracy of the individual model (near-infrared light transillumination [NILT] device, visual and radiographic examinations) in detecting occlusal caries, and to evaluate the performance of visual and NILT device combination for occlusal caries detection in deciding the treatment options. Fifty-two non-cavitated occlusal surfaces from 16 patients were assessed with three different diagnostic devices in random order. Identified lesions were prepared and validated. Logistic regression analysis was performed for each method. The sensitivity and specificity values for each method and the combined models were statistically measured using RStudio version 0.97.551. At the enamel level, visual detection was the most sensitive method $(0.88)$, while NILT was the most specific (0.93). NILT scored the highest for sensitivity (0.93) at the dentine level and visual detection scored the highest for specificity (0.88). Visual detection + NILT model was significantly better $(p=0.04)$ compared to visual detection or NILT alone $(d f=1)$. The visual-NILT combination is a superior model in detecting occlusal caries on permanent teeth. The model provided surplus value in caries detection hence improving the treatment decision-making in occlusal surfaces.

Keywords: Diagnostic imaging; near-infrared light transillumination; occlusal caries; paediatric; permanent teeth 


\section{INTRODUCTION}

There had been a parallel effort to detect caries lesion as early as possible even before the non-cavitated stage along with the development of a quantitative method for caries lesion detection (Pitts, 2004). This effort has been driven by the acceptance that early identification provides better estimations of the disease and improves the possibility for successful preventive intervention. However, from a clinical perspective, this is an arguable statement as a low diagnostic threshold may lead to the detection of discoloured surfaces, thus increasing false-positive diagnosis (Nyvad, 2004). On the other hand, the creation of the International Caries Detection and Assessment System (ICDAS) in 2002 strengthens the idea of early detection of caries lesion. ICDAS shows a significant correlation with lesion depth in the histological examination (Shoaib et al., 2009; Gomez, Tellez et al., 2013). It has shown to be an accurate and reproducible method to detect early lesions and changes during longitudinal follow-up (Braga et al., 2009). The use of bitewing radiographs provides supportive information to complete the clinical diagnosis in proximal and occlusal tooth surfaces (Hintze et al., 1998; Espelid et al., 2003; Schaefer et al., 2018).

The range of caries diagnosis options has expanded considerably in recent years, especially in X-ray free diagnostics. The application of various technologies provides clinicians with affordable options, some of which prove to be equal or superior to radiography. The objective should be to detect carious lesions in the initial stage with optimum efficiency. A variety of non-invasive technologies for incipient, intermediate and hidden caries detection have been investigated and made available such as fibre-optic transillumination (FOTI), laser fluorescence (LF) and electrical conductance (EC) (Yang \& Dutra, 2005; Gomez, Tellez et al., 2013; Tassery et al., 2013; Schaefer et al., 2018). Most of the published studies on the detection systems mentioned above have shown acceptable performance and reproducibility for the detection and quantification of dentinal lesions (Gomez, Zakian et al., 2013), incipient and hidden caries (Gomez, Tellez et al., 2013), early and intermediate occlusal caries (Gomez, Zakian et al., 2013), and monitoring the disease in assessing the efficacy of anti-caries dental care products (Gomez, 2015). Each performance of clinical testing is evaluated in terms of specificity and sensitivity to ensure the method effectiveness.

In the context of caries, sensitivity refers to the test's ability to identify evidence of caries formation correctly. In contrast, specificity refers to the ability to rule out caries correctly or to identify surfaces that are non-caries (Martin, 2007). Although it was evident that both properties should be maximised in a test, an increase in one means a decrease in the other and vice versa.

Transillumination with light has been further developed into cold visible light (Wenzel et al., 1992; Schneiderman et al., 1997; Young \& Featherstone, 2005). This technique allows images from tooth surfaces to be digitally captured and stored. Moreover, it has made this technology accessible for practitioners and sophisticated enough for longitudinal evaluation of individual caries lesions. The package's special software allowed the diagnosis to be made on a computer screen and visualised by a live video. The device established a good agreement with the clinical status and information beyond the radiograph (Bin-Shuwaish et al., 2008) and can be useful in detecting incipient and hidden carious lesions (Yang \& Dutra, 2005). The DIAGNOcam $^{\circledR}$ is the most recent developed near-infrared light transillumination (NILT) device $\left(\mathrm{KaVo}^{\circledR}\right.$ Dental, Lake Zurich, IL, United States). It is a fibre-optic transillumination (excitation wavelength of $780 \mathrm{~nm}$ ) device with a camera. The device is placed directly on the tooth and the transilluminating light positioned on either side of the tooth shines through the tooth. A video camera captures the light where a 
carious lesion appears as dark spots. The software allows storage of the images.

A previous in vivo diagnostic study had shown signifying correlation of utilising visual inspection, bitewing radiographs and NILT as diagnostic strategies to avoid over-diagnosis (Schaefer et al., 2018). Additionally, NILT is shown as suitable for detecting early caries lesion (Elhennawy et al., 2018). There are numerous reports on ICDAS studies, but there were only a few DIAGNOcam ${ }^{\circledR}$ studies that focused in detecting the occlusal caries (MarinovaTakorova et al., 2016; Simon et al., 2017; Schaefer et al., 2018; Tassoker et al., 2020).

Therefore, the aims of the present in vivo study were twofold: (1) To assess the accuracy of the individual model (NILT device, visual and radiographic examinations) in detecting occlusal caries and (2) To evaluate the performance of visual and NILT device combination for occlusal caries detection in deciding treatment options.

\section{MATERIALS AND METHODS}

\section{Study Design and Sampling}

For the present in vivo study, the proposed minimum sample size required for diagnostic values (prevalence of $30 \%, p$ value 0.05 and power of $80 \%$ ) was 31 samples for sensitivity test and 13 samples for specificity test (Bujang \& Adnan, 2016). The study engaged 16 new patients (7-15 years old) with informed verbal and written consent from October 2016 to March 2017 who attended the Paediatric Dental Clinic. The methods were tested on 52 occlusal surfaces of identified molars or premolars. The patients had or would have had at least one fully erupted first permanent molar and/or premolars with ICDAS 1 (first visual change in enamel) or ICDAS 2 (distinct visual change in enamel). All patients recruited were diagnosed with high caries risk based on the medical model of risk for dental caries (Kutsch, 2014). Exclusions were made for cases that exhibited uncooperative patients, special needs children, teeth with apparent dentine caries (ICDAS 3, 4 and 5), enamel/ dentine anomalies, smooth surface caries (based on a visual inspection and bitewing radiograph) and any other types of teeth restorations. All procedures performed in this study were approved by the Ethical Committee of Research Management Institute (RMI), Universiti Teknologi MARA (UiTM) (600-IRMI (5/1/6) REC/115/17). The study was carried out in accordance with the Declaration of Helsinki 1964. Informed consent was obtained from each of the individual participants included in the study.

\section{Examiners' Calibration and Validation}

A total of three calibrated examiners with a minimum of five years of professional qualification and experience took part in the validation process. Furthermore, all examiners were calibrated for studied detections methods; Visual-ICDAS photographs, digital bitewing radiographs and NILT images. Data was presented in Microsoft PowerPoint version 2003 (Microsoft Corporation, New Mexico, United States) for the evaluation process. The evaluation was carried out according to an agreed environment setting and procedure of conduct by all evaluators. A total of 10 photographs and images were evaluated and scored based on the criteria described further in the article. The intra/inter-examiner reproducibility was expressed by the kappa statistic, where 10 non-cavitated, discoloured molar and/premolar surfaces were examined in the mean of ICDAS, NILT and bitewing radiographs scores and repeated two weeks later. The intra- and inter-examiner reproducibility interpretation was calculated based on previous studies (Landis \& Koch, 1977; McHugh, 2012).

The newly developed NILT scores were assembled and validated by all three calibrated examiners. The scores were defined based on the device image shadow extension, broadening and intensity. It had been divided into five scores (0 to 4 ), 
translating into the sound surface, two dissimilar surface levels of enamel involvement and two distance surface levels of dentine involvement.

The study involved two stages of scoring. The initial stage consisted of scoring the records according to the defined criteria. The second stage was to validate operator's treatment based on clinical photographs, bitewings radiograph and NILT images, and a combination of clinical photographs and NILT images. The included lesions were validated as the reference standard.

\section{Examination Methods}

\section{Visual Inspection}

The principal investigator (IWM) was the sole operator for all the examinations process. Possible confounding factors such as plaque or calculus were eliminated using strict inclusion criteria and consistent cleaning procedure of the occlusal surfaces. All selected occlusal surface sites were brushed using a rotary brush and slow handpiece, rinsed (5 seconds), air-dried (5 seconds) and visually examined using a dental light and mirror. The occlusal surfaces of the teeth were photographed using Nikon D90 (Nikon Corporation, Thailand). The region of interest (ROI) was virtually outlined using the groove-fossa system. The basis of validation was topographical position and enamel spectrum changes on the occlusal surface of a tooth. ICDAS was used for the scoring (Ismail et al., 2007) (see Table 1). These were labelled as the model of the Visual-ICDAS photograph.

\section{Bitewing Radiography}

Bitewing radiograph was performed using CMOS sensor (EzSensor, pixel size $35 \mu \mathrm{m}$, Vatech, Hwaseong, Korea) with plastic bitewing holder (Kwik-bite, Kerr United Kingdom) coupled to an intraoral machine (Satelec X-Mind AC/DC, Satelec ACTEON Tuusula, Finland). The system automatically registered all digital radiographic examinations. The images were collected and evaluated on EasyDent V4 ${ }^{\circ}$ viewer software version 4.1.4.5 (Vatech, Hwaseong, Korea). The radiographs classification was adopted from a previous study (Anttonen et al., 2003) (see Table 2). This method was designated as the digital radiographs model.

Table 1 ICDAS scores (as adopted from Ismail et al., 2007)

\begin{tabular}{cl} 
Score & \multicolumn{1}{c}{ Visual (ICDAS) } \\
0 & No or slight change in enamel translucency after prolonged air drying. \\
1 & First visual change in enamel (after drying) or restricted to within the confines of a pit or fissures. \\
2 & Distinct visual change in enamel (on wet surface). \\
3 & Localised enamel breakdown in opaque or discoloured enamel without visual change of dentinal involvement. \\
4 & Underlying dark shadow from dentin with or without localised enamel breakdown. \\
\hline
\end{tabular}

Table 2 Digital bitewing radiographs scores (as adopted from Anttonen et al., 2003)

\begin{tabular}{cl} 
Score & \multicolumn{1}{c}{ Digital radiograph } \\
\hline 0 & No radiolucency. \\
1 & Radiolucency of the enamel. \\
2 & Radiolucency extending to enamel-dentine junction or superficial dentine. \\
3 & Radiolucency extending to the middle third of dentine. \\
4 & Radiolucency extending to deep inner third of dentine. \\
\hline
\end{tabular}




\section{NILT Procedure}

The NILT investigation was carried out using DIAGNOcam ${ }^{\circledR} \quad\left(\mathrm{KaVo}^{\circledR} \quad\right.$ Dental $\mathrm{GmbH}$, Germany), where the camera arm was placed as perpendicular as possible to embrace most of the occlusal anatomy surface. The digital images captured were labelled and recorded accordingly. A newly developed and validated score was used to rank the NILT images (Table 3). This method was categorised as the NILT images model.

\section{Clinical Validation Criteria}

Based on the ICDAS, the surface discolouration was evaluated and scored as 1 or 2 ; both were treated either with topical fluoride, fissure sealant or fissure sealant applied through enameloplasty in caries-active individuals (Smallridge et al., 2000; Bader et al., 2001; Deery, 2013). The selection of patients was retrieved from the Integrated Dental Record and Management System (Universiti Teknologi MARA, Malaysia) where operators indicated each patient's treatment needs, based on their professional assessment after taking into account the individual's caries risk assessment. Later, the primary and second researcher (IWM and APV) validated the treatment selection and decided based on the binary presence or absence of caries lesion. Afterwards, they performed the chosen treatment procedure accordingly. Fissures were cleaned, prepared and assessed. Surfaces with discolouration that did not invade beyond the fissure system would be sealed using preventive resin sealant, and the tooth was classified as enamel caries and labelled as (E). Based on the newly developed NILT scores in Table 3, enameloplasty was proposed on the surfaces which scored 1 to 4 and not on the sound surfaces, which scored 0 . Surfaces with deeper demineralisation at the occlusal surface (scores 3 and 4) dictated the preparation of cavity using a small round bur diamond (size 8), medium (size 10) or large (size 12) as necessary to remove the undermined or/and overhanging enamel to allow self-cleansing of the lesion. The prepared cavity then was observed for the lesion's soft surface layer became worn away, leaving a hard and pigmented surface (Deery, 2013). The cavity later was warranted as caries-free through visual inspection; the tooth would be classified as dentine caries and labelled as (D).

\section{Statistical Analysis}

Data were analysed using RStudio version 0.97.551 ${ }^{\circledR} 2009-2012$ Inc. software (RStudio, PBC, Boston, MA). Intra- and inter-examiner values for all detection models were calculated using a weighted kappa statistic. Visual examination after tooth preparation served as the standard reference in calculating sensitivity and specificity. The enamel and dentine caries were used as the binary response, while Visual-ICDAS photographs, NILT images and digital radiographs were used as predictors. Logistic regression analysis was performed for each model. A likelihood ratio test (LRT) was used to compare nested models. This test yields chi-square $\left(\chi^{2}\right)$

Table 3 Newly developed NILT scores

\begin{tabular}{cl} 
Score & \\
\hline 0 & Normal shadow of fissures. \\
1 & Continuous pencil thin-line, less intense grey shadow appears when transilluminated. \\
2 & Broadening of the shadow restricted to the fissure, more intense grey shadow appears when transilluminated. \\
3 & Wide, dark-greyish and expecting dentine perforation shadow appears when transilluminated. \\
4 & $\begin{array}{l}\text { Wide, dark-greyish shadow and expecting dentine perforation includes groove-fossa cusp system appears } \\
\text { when transilluminated. }\end{array}$
\end{tabular}


value and a $p$ value. If two nested models are compared and the LRT produces $p \leq 0.05$, the model being compared fits the data better than the base model. The area under receiver operating characteristic (AUROC) was calculated at the enamel and dentine threshold level. The optimum performance of each model's threshold values for enamel and dentine were determined from the AUROC analysis. The optimum value was defined by the visual measure of the predictive ability of the logistic regression model. The optimal cutoff points corresponding to the maximum combination sensitivity and specificity observed were dichotomised into two cutoffs: $0=$ enamel and $1=$ dentine.

\section{Image Investigation and Assessment}

Fig. 1 illustrates the Visual-ICDAS photographs, NILT images and bitewing radiographs of different demineralisation degree of enamel and occlusal caries. The images represent the stages of caries invasion and extension starting from; (a) sound surface with discoloured fissure, (b) early enamel caries, (c) discoloured fissures of the groove-fossa system with dentine penetration and finally (d) obvious dentine involvement.

(a)

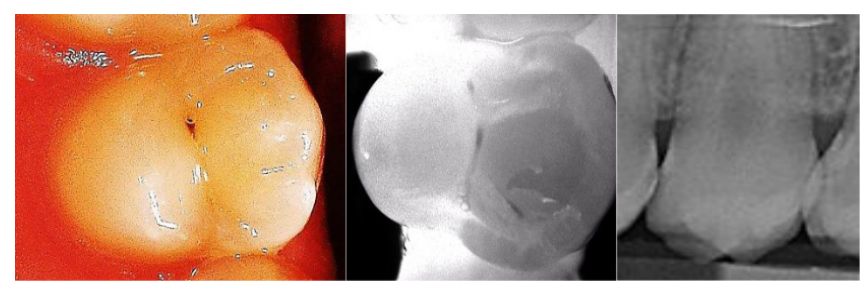

(b)

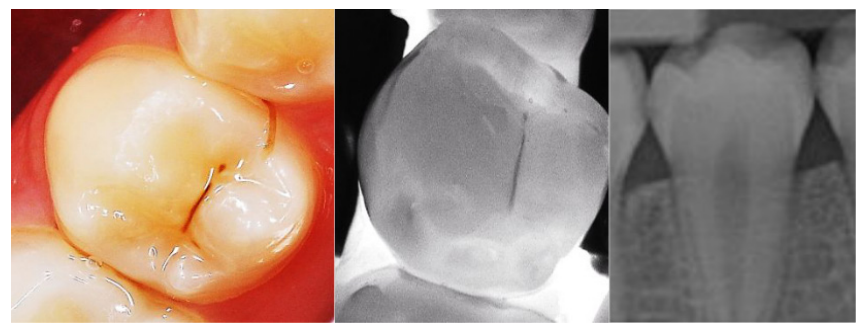

(c)

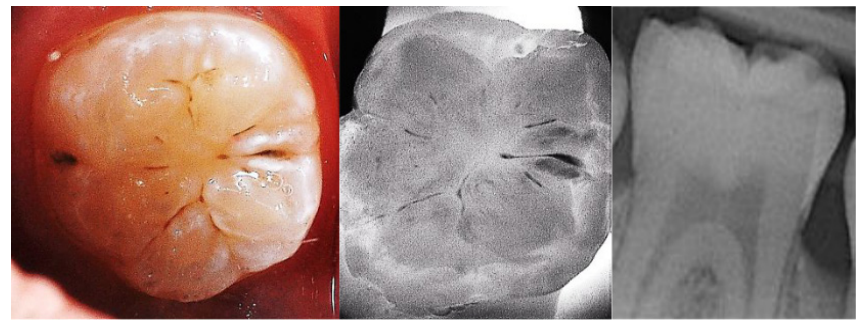

(d)

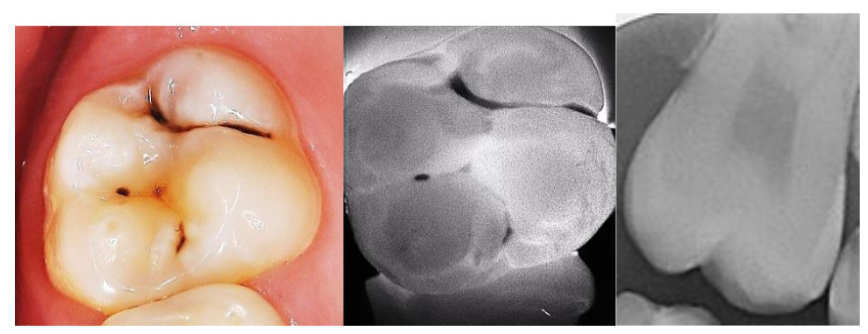

Fig. 1 The visual-ICDAS photographs, NILT digital images and bitewing radiographs of different demineralisation degree of enamel and occlusal caries. The images represent the stages of caries invasion and extension starting from (a) sound surface with discoloured fissure, (b) early enamel caries, (c) discoloured fissures of the groove-fossa system with dentine penetration and finally (d) obvious dentine involvement. 


\section{RESULTS}

A total of 52 teeth (16 children) of 44 molars and eight premolars were examined and treated accordingly. When conferred to the standard reference, 28 teeth had caries into the dentine and restored with resin material. In comparison, 24 teeth had caries restricted to the enamel and sealed with preventive resin restoration. The gender distribution was equal with eight female and eight male children. The intra-examiner reproducibility (weighted kappa) was as follows; 0.85 (IWM), 0.91 (APV) and 0.93 (MYPMY) for Visual-ICDAS, 0.91 (IWM), 0.89 (APV) and 0.90 (MYPMY) for NILT, 0.83 (IWM), 0.91 (APV), 0.93 (MYPMY) for bitewing. Accordingly, the inter-examiner reproducibility (weighted kappa) of NILT examinations between primary examiner (IWM) and experienced examiner one (APV) and two (MYPMY) were 0.92, 0.95 respectively and 0.72 for all three evaluators. For reliability interpretation, the present study's weighted kappa values were based on previous studies (Landis \& Koch, 1977; McHugh, 2012). In general, all kappa values were varied between the substantial (61-80) and had an almost perfect agreement (81100).

Table 4 shows the correlation between cutoffs, sensitivity, specificity and AUROC at enamel and dentine threshold level. The AUROC curves scaled 0.90 (Visual-ICDAS), 0.93 (NILT), 0.73 (digital radiograph) and 0.98 (combination model Visual-NILT) to discriminate the caries detection either in enamel or in dentine (Fig. 2). Sensitivities on enamel level varied between 0.88 (VisualICDAS), 0.83 (NILT) and 0.83 (digital radiograph); specificities varied from 0.89 (Visual-ICDAS), 0.93 (NILT) and 0.61 (digital radiograph). At the dentine level, sensitivity ranged between 0.89 (VisualICDAS), 0.93 (NILT) and 0.61 (digital radiograph) and specificity from 0.88 (Visual-ICDAS), 0.83 (NILT) and 0.83 (digital radiograph). The study expressed that visual detection was the most sensitive method (0.88), while NILT was the most specific (0.93). NILT scored the highest for sensitivity (0.93) at the dentine level and visual detection scored the highest for specificity (0.88). On the other hand, the combination-model Visual-NILT method reported a sensitivity of 0.92 and specificity of 0.93 at the enamel level. The model also reported high sensitivity and specificity at the dentine level with 0.93 and 0.92, respectively. The full logistic regression model of Visual-ICDAS and NILT was statistically significant $(p \leq 0.05)$ compared to the reduced model of both VisualICDAS and NILT separately $(d f=1)$. This finding suggested that Visual-NILT was a better model in detecting occlusal caries on permanent teeth (Fig. 3).

\section{DISCUSSION}

Early detection of occlusal caries plays a significant role in dental practice and minimally invasive management. Since the 1990s, clinical trials worldwide have provided evidence that clinically diagnosed caries lesions, at the non-cavitated stage,

Table 4 Sensitivity, specificity and AUROC between the caries detection methods

\begin{tabular}{|c|c|c|c|c|c|}
\hline \multirow{3}{*}{ Methods } & \multicolumn{5}{|c|}{ Cut-off points } \\
\hline & \multicolumn{2}{|c|}{ Enamel } & \multicolumn{2}{|c|}{ Dentine } & \multirow{2}{*}{$\begin{array}{l}\text { AUROC ( } 98 \% \mathrm{CI}) \\
\text { Enamel/Dentine }\end{array}$} \\
\hline & Sensitivity & Specificity & Sensitivity & Specificity & \\
\hline Visual (ICDAS photograph) & 0.88 & 0.89 & 0.89 & 0.88 & $0.90(0.82 ; 1.00)$ \\
\hline DIFOTI (DIAGNOcam) & 0.83 & 0.93 & 0.93 & 0.83 & $0.93(0.86 ; 0.99)$ \\
\hline Digital radiograph (Bitewings) & 0.83 & 0.61 & 0.61 & 0.83 & $0.73(0.60 ; 0.87)$ \\
\hline $\begin{array}{l}\text { Combination model visual-ICDAS } \\
\text { and DIFOTI }\end{array}$ & 0.92 & 0.93 & 0.93 & 0.92 & $0.92(0.90 ; 0.98)$ \\
\hline
\end{tabular}




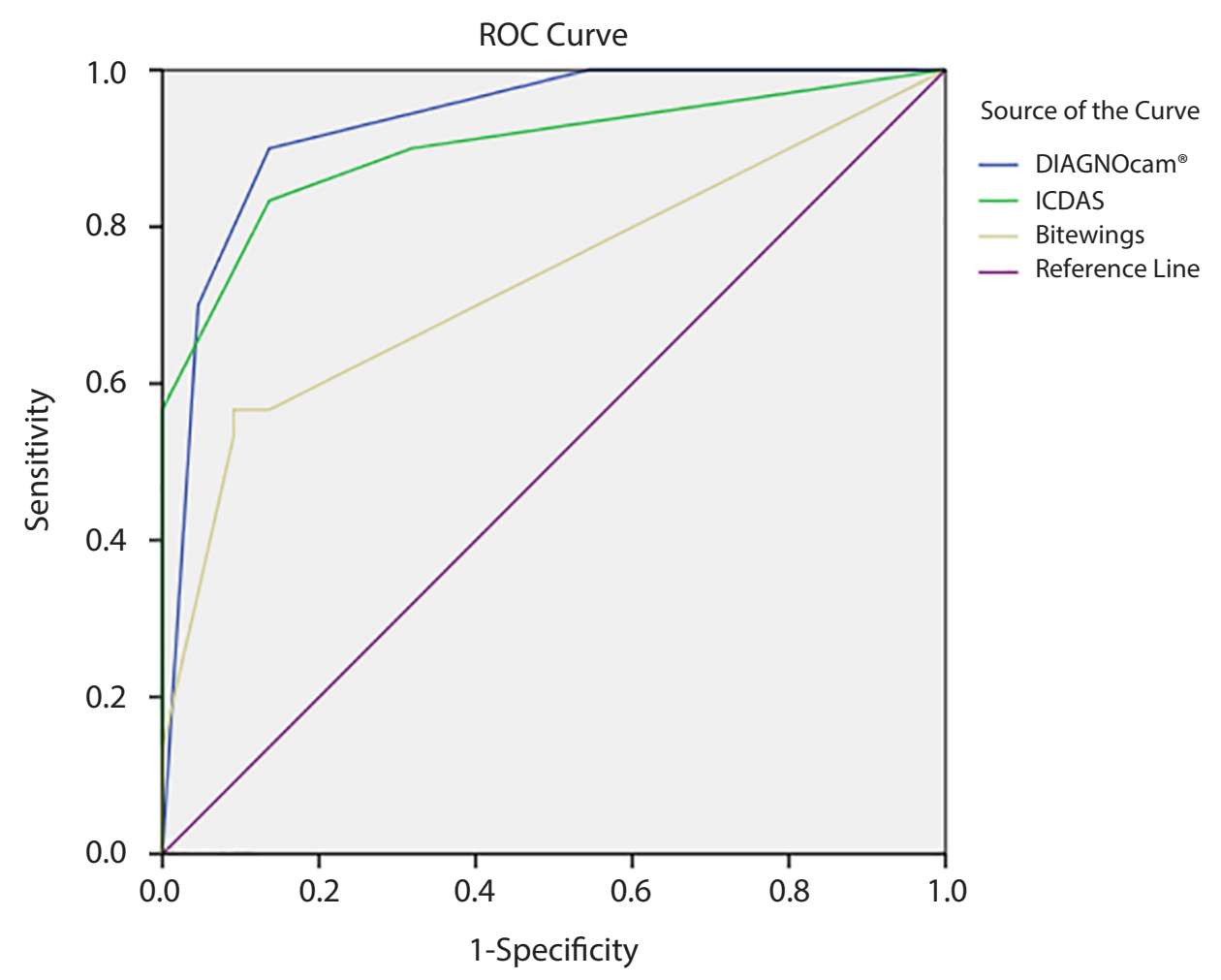

Fig. 2 The area under ROC curve for all three predictors.

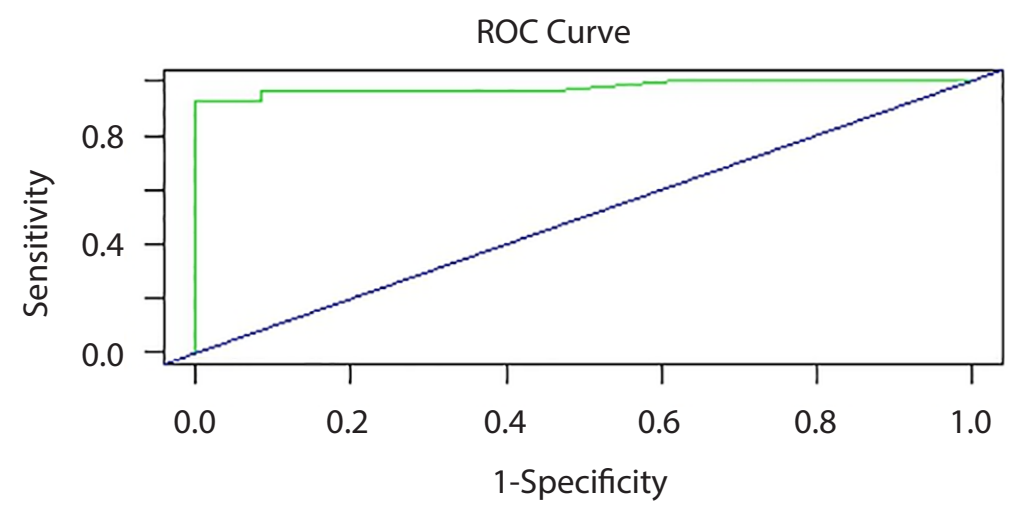

Fig. 3 The area under the ROC curve for the full model (visual-ICDAS and NILT images).

in particular, may be successfully treated by non-operative strategies (Carvalho et al., 1992; Machiulskiene et al., 2001, 2002; Maltz et al., 2003; Marinho et al., 2003; Ismail et al., 2013). However, when the diagnostic process is related to management strategies, it becomes a major challenge for dental professionals (Machiulskiene \& Carvalho, 2018). Moreover, there is a need to detect or quantitatively monitor such lesions to support treatment decisions.
The narrow and deep fissure system increased the susceptibility to occlusal caries in children and young adolescents and should regularly be inspected for caries signs (Dodds, 1996; Muller-Bolla et al., 2009). Visual inspection may underestimate occlusal dentin caries in teeth without cavitation (Lussi, 1993; Gimenez et al., 2015). Moreover, there is a need to detect or quantitatively monitor such lesions to support treatment decisions. The present 
sample consists of children in their mixed dentition with at least one discoloured pitfissure system of first permanent molar and premolar. During this post-eruptive period, the tooth is most susceptible to caries. Thus, all samples were entitled to a preventive sealing or preventive resin restoration concerning the patient's individual caries risk assessment and caries history. All the tooth surfaces scored 1-4 had undergone enameloplasty which revealed the high sensitivity of visual examination in discriminating the lesion extension in the enamel.

The intra- and inter-examiner reliability revealed substantial to an almost perfect agreement for all the performed methods. Specifically, for the DIAGNOcam ${ }^{\circledR}$ device, obtaining reproducibility was very promising even though all the examiners had different experience lengths. Nevertheless, the practitioners tend to develop individual concepts based on experience regarding caries detection and the subsequent preventive or restorative treatment options. Therefore, a learning curve in using the device must be taken into consideration. Schneiderman et al. (1997) concluded that the real-time analysis images enable the practitioners to obtain NILT images under controlled and repeatable conditions. This value contributed significantly to the reproducibility properties of the device.

A valuable feature of quantitative caries diagnostic tools is the possibility to establish cut-off levels to determine caries penetration (Huth et al., 2008). In the perspective of this in vivo study, the cut-off points for lesion depths were defined as enamel or dentine based on the visual examination. The distinction between the enamel and dentine level cannot be quantified entirely using DIAGNOcam ${ }^{\circledR}$. Such findings were in concordance with the other previous studies (Pretty \& Maupome, 2004; Schaefer et al., 2018). Since the captured images are not directly quantified and the clinician must perform the analysis within all the advantages and disadvantages that such analyses require, a learning curve is expected in mastering the device (Sidi \& Naylor, 1988).

The results demonstrated a potential significance of the NILT (DIAGNOcam ${ }^{\circledR}$ ) model for detecting occlusal caries with high sensitivity to conventional models. The NILT images indicated early incipient caries on the discoloured groove-fossa system even when digital radiograph images failed to show their presence and that NILT provided clear signatures of occlusal caries on premolars and molars.

The principle behind a tooth's transillumination is that demineralised areas of enamel or dentine scatter light more than sound areas (Angelino et al., 2017). Thus, the NILT captured images were interpreted as follows:

1. The darker the shadows of light projected onto the tooth surface, the deeper caries invasion to be anticipated.

2. If the shadows were estimated to be beyond the groove-fossa system of the fissure, the widening/broadening of the cavitation was presumed, and therefore more chances of dentine perforation could be expected.

The above criteria used were correlated with one of the earliest in vitro study on near-IR imaging performance (Staninec et al., 2010). The detection was significantly based on subjective interpretation of the lesion appears as such. On the other hand, an occlusal surface with deep fissures with no demineralised activity detected visually might be projected by a fine line shadow and possibly considered as demineralised surfaces. This restriction might contribute to the increase in the over-scoring tendency of discoloured sites.

The findings described in Table 4 show that DIAGNOcam ${ }^{\circledR}$ performed best when observing the dentine caries and to detect no caries in the enamel surface. The diagnostic performance established by the receiver 
operating characteristic (ROC) curve of both Visual-ICDAS and NILT images gave an almost perfect result. Bitewing radiography performance was found to be in substantial agreement with the samples. However, when compared with the other two detection methods, it might be giving the lowest efficiency in detecting the occlusal caries. The variability in sensitivity is high, compared to specificity when occlusal surfaces with different depths of the lesion was evaluated using a bitewing radiograph (Dove, 2001).

In this study, the sensitivity values for Visual-ICDAS could have been influenced negatively due to the assessment performed using images instead of direct in vivo scoring. The quality of the taken photographs of the occlusal surface can also be debatable. Although enamel caries cannot be validated in a clinical study (Anttonen et al., 2003) and the present study, the images of V-NILT suggest that an experienced and well-trained clinician can differentiate between enamel or dentine caries in the pit-fissures system. The NILT images can considerably contribute to making the diagnosis and treatment decision. The present study adopted the bitewing radiograph scores from another in vivo study by Anttonen et al. (2003) because it resembles similarity in nature with our investigated lesion.

The NILT images obtained indicated equal low sensitivity in the enamel level and specificity in dentine level when compared to bitewing radiography. However, the anatomic configuration of the occlusal surface also explaining the low sensitivity in bitewing radiographs. The sensitivity in dentine level and specificity in enamel level, however, were higher. This finding is also supported by a previous study (Sidi \& Naylor, 1988) that compared visual inspection with FOTI and various image modalities, suggesting superior sensitivity of FOTI compared with radiological imaging. These findings may be explained by many different factors that can affect the ability to accurately detect these lesions such as exposure parameters, type of image receptor, image processing, display system, viewing conditions, and eventually the training and experience of the human observer (Dove, 2001). Despite the low dose of radiation exposure from taking bitewing radiographs in caries detection, according to the linearno-threshold nature of radiation effect, theoretically, there is no safe dose in radiographic acquisition (Yusof et al., 2017).

The NILT images exhibit lesion extension in horizontal plane angle surface but there is no substantiation of lesion activity towards the pulpal region. The caries invasion depth cannot be determined with the device since the light transilluminate only in twodimensional (2D) perspectives. Hence the combination with a Visual-ICDAS method which has been validated based on the quantitative correlation between the clinical assessments of tooth surfaces with the histological presence or extent of demineralisation in enamel and dentin (Ekstrand et al., 1997; Ricketts et al., 2002) possibly will aid to overcome this setback.

Detection of a lesion at a very early stage requires a diagnostic method with high sensitivity. However, that may result in more false-positive diagnoses (Kühnisch et al., 2006) and lower specificity. Due to the lack of a single detection method that provides high sensitivity and high specificity, combining some methods is recommended to increase detection accuracy. We found that the combination method resulted in an almost perfect sensitivity and specificity value on enamel and dentine levels. The proposed model, namely the Visual-NILT, is superior in differentiating caries lesions in the inner half of enamel from those in the outer third of dentine (Côrtes et al., 2003) and is effective in the diagnosis of lesion both involving only the enamel as well as enameldentine (Marinova-Takorova et al., 2016). Therefore, the combination method would facilitate treatment decision-making, thereby preventing overtreatment. 


\section{CONCLUSION}

The NILT presented a similar efficiency to visual inspection and was superior compared to bitewing radiography. The NILT device can provide satisfactory information for the diagnosis and treatment selection of occlusal caries, and it has significantly adds value when combined with a visual inspection. Moreover, this method may avoid the use of bitewing radiography, minimising the chance of radiation exposure of the children, thus increasing safe practice.

\section{ACKNOWLEDGEMENTS}

The authors would like to express gratitude to all staff members of Paediatrics Dentistry Clinic, Faculty of Dentistry, Universiti Teknologi MARA and the Ministry of Higher Education Malaysia for the kind support.

\section{REFERENCES}

Angelino K, Edlund DA, Shah P (2017). Nearinfrared imaging for detecting caries and structural deformities in teeth. IEEE f Transl Eng Health Med, 5: 2300107. https://doi.org/10.1109/JTEHM.2017 .2695194

Anttonen V, Seppä L, Hausen H (2003). Clinical study of the use of the laser fluorescence device DIAGNOdent for detection of occlusal caries in children. Caries Res, 37(1): 17-23. https://doi.org/10 $.1159 / 000068227$

Bader JD, Shugars DA, Bonito AJ (2001). Systematic reviews of selected dental caries diagnostic and management methods. f Dent Educ, 65(10): 960-968.

Bin-Shuwaish M, Yaman P, Dennison J, Neiva G (2008). The correlation of DIFOTI to clinical and radiographic images in Class II carious lesions. I Am Dent Assoc, 139(10): 1374-1381. https://doi.org/10.14219/jada. archive.2008.0049
Braga MM, Mendes FM, Martignon S, Ricketts DN, Ekstrand KR (2009). In vitro comparison of Nyvad's system and ICDAS-II with lesion activity assessment for evaluation of severity and activity of occlusal caries lesions in primary teeth. Caries Res, 43(5): 405-412. https://doi.org/ $10.1159 / 000239755$

Bujang MA, Adnan TH (2016). Requirements for minimum sample size for sensitivity and specificity analysis. F Clin Diagn Res, 10(10): YE01-YE06. https://doi.org/10 .7860/JCDR/2016/18129.8744

Carvalho JC, Thylstrup A, Ekstrand KR (1992). Results after 3 years of non-operative occlusal caries treatment of erupting permanent first molars. Community Dent Oral Epidemiol, 20(4): 187-192. https://doi.org/10.1111/j.1600-0528.1992 .tb01713.x

Côrtes DF, Ellwood RP, Ekstrand KR (2003). An in vitro comparison of a combined FOTI/visual examination of occlusal caries with other caries diagnostic methods and the effect of stain on their diagnostic performance. Caries Res, 37(1): 8-16. https://doi.org/10.1159/000068230

Deery C (2013). Caries detection and diagnosis, sealants and management of the possibly carious fissure. $\mathrm{Br}$ Dent $\mathcal{F}, \quad 214(11)$ : 551-557. https://doi.org/10.1038/sj.bdj .2013 .525

Dodds MW (1996). Dental caries diagnosis toward the 21st century. Nat Med, 2(3): 283. https://doi.org/10.1038/nm0396-283

Dove SB (2001). Radiographic diagnosis of dental caries. I Dent Educ, 65(10): 985990.

Ekstrand KR, Ricketts DN, Kidd EA (1997). Reproducibility and accuracy of three methods for assessment of demineralization depth of the occlusal surface: An in vitro examination. Caries Res, 31(3): 224-231. https://doi.org/10.1159/000262404 
Elhennawy K, Askar H, Jost-Brinkmann PG, Reda S, Al-Abdi A, Paris S et al. (2018). In vitro performance of the DIAGNOcam for detecting proximal carious lesions adjacent to composite restorations. F Dent, 72: 39-43. https://doi.org/10.1016/j.jdent .2018 .03 .002

Espelid I, Mejàre I, Weerheijm K (2003). EAPD guidelines for the use of radiographs in children. Eur $\mathcal{F}$ Paediatr Dent, 4(1): 40-48.

Gimenez T, Piovesan C, Braga MM, Raggio DP, Deery C, Ricketts DN et al. (2015). Visual inspection for caries detection: A systematic review and meta-analysis. f Dent Res, 94(7): 895-904. https://doi .org/10.1177/0022034515586763

Gomez J (2015). Detection and diagnosis of the early caries lesion. BMC Oral Health, 15(Suppl 1): S3. https://doi.org/ 10.1186/1472-6831-15-S1-S3

Gomez J, Tellez M, Pretty IA, Ellwood RP, Ismail AI (2013). Non-cavitated carious lesions detection methods: A systematic review. Community Dent Oral Epidemiol, 41(1): 54-66. https://doi.org/10.1111/cdoe .12021

Gomez J, Zakian C, Salsone S, Pinto SC, Taylor A, Pretty IA et al. (2013). In vitro performance of different methods in detecting occlusal caries lesions. $\mathcal{F}$ Dent, 41(2): 180-186. https://doi.org/10.1016/ j.jdent.2012.11.003

Hintze H, Wenzel A, Danielsen B, Nyvad B (1998). Reliability of visual examination, fibre-optic transillumination, and bitewing radiographs, and reproducibility of direct visual examination following tooth separation for the identification of cavitated carious lesions in contacting approximal surfaces. Caries Res, 32(3): 204-209. https://doi.org/10.1159/000016454
Huth KC, Neuhaus KW, Gygax M, Bücher K, Crispin A, Paschos E et al. (2008). Clinical performance of a new laser fluorescence device for detection of occlusal caries lesions in permanent molars. $f$ Dent, 36(12): 1033-1040. https://doi.org/10 $.1016 /$ j.jdent.2008.08.013

Ismail AI, Sohn W, Tellez M, Amaya A, Sen A, Hasson $\mathrm{H}$ et al. (2007). The International Caries Detection and Assessment System (ICDAS): An integrated system for measuring dental caries. Community Dent Oral Epidemiol, 35(3): 170-178. https://doi.org/10.1111/j.1600-0528.2007 $.00347 . \mathrm{x}$

Ismail AI, Tellez M, Pitts NB, Ekstrand KR, Ricketts D, Longbottom C et al. (2013). Caries management pathways preserve dental tissues and promote oral health. Community Dent Oral Epidemiol, 41(1): e12-e40. https://doi.org/10.1111/cdoe .12024

Kühnisch J, Heinrich-Weltzien R, Tabatabaie M, Stosser L, Huysmans MC (2006). An in vitro comparison between two methods of electrical resistance measurement for occlusal caries detection. Caries Res, 40(2): 104-111. https://doi .org/10.1159/000091055

Kutsch VK (2014). Dental caries: An updated medical model of risk assessment. f Prosthet Dent, 111(4): 280-285. https://doi.org/10.1016/j.prosdent.2013.07 .014

Landis JR, Koch GG (1977). The measurement of observer agreement for categorical data. Biometrics, 33(1): 159-174. https://doi.org/ $10.2307 / 2529310$

Lussi A (1993). Comparison of different methods for the diagnosis of fissure caries without cavitation. Caries Res, 27(5): 409-416. https://doi.org/10.1159/000261572 
Machiulskiene V, Carvalho JC (2018). Clinical diagnosis of dental caries in the $21 \mathrm{st}$ century: Introductory Paper - ORCA Saturday Afternoon Symposium, 2016. Caries Res, 52(5): 387-391. https://doi.org/ $10.1159 / 000486430$

Machiulskiene V, Nyvad B, Baelum V (2001). Caries preventive effect of sugar-substituted chewing gum. Community Dent Oral Epidemiol, 29(4): 278-288. https://doi.org/ 10.1034/j.1600-0528.2001.290407.x

Machiulskiene V, Richards A, Nyvad B, Baelum V (2002). Prospective study of the effect of postbrushing rinsing behaviour on dental caries. Caries Res, 36(5): 301-307. https://doi.org/10.1159/000065955

Maltz M, Barbachan e Silva B, Carvalho DQ, Volkweis A (2003). Results after two years of non-operative treatment of occlusal surface in children with high caries prevalence. Braz Dent F, 14(1): 48-54. https://doi.org/10.1590/s0103-644020030 00100009

Marinho VC, Higgins JP, Sheiham A, Logan $S$ (2003). Fluoride toothpastes for preventing dental caries in children and adolescents. Cochrane Database Syst Rev, (1): CD002278. https://doi.org/10.1002/ 14651858.CD002278

Marinova-Takorova M, Panov V, Anastasova $R$ (2016). Effectiveness of near-infrared transillumination in early caries diagnosis. Biotechnol Biotechnol Equip, 30(6): 12071211. https://doi.org/10.1080/13102818 .2016 .1232606

Martin C (2007). Epidemiology, biostatistics, and surveillance. In: Markle WH, Fisher MA, Smego RA Jr (eds.), Understanding Global Health. New York: Mc Graw Hill Lange. pp. 37-60.

McHugh ML (2012). Interrater reliability: The kappa statistic. Biochem Med (Zagreb), 22(3): 276-282.
Muller-Bolla M, Courson F, Droz D, LupiPégurier L, Velly AM (2009). Definition of at-risk occlusal surfaces of permanent molars-A descriptive study. $\mathcal{f}$ Clin Pediatr Dent, 34(1): 35-42. https://doi.org/ 10.17796/jcpd.34.1.n7r85u5788575766

Nyvad B (2004). Diagnosis versus detection of caries. Caries Res, 38(3): 192-198. https://doi.org/10.1159/000077754

Pitts NB (2004). Modern concepts of caries measurement. F Dent Res, 83(Spec No C): C43-C47. https://doi.org/10.1177/15440 5910408301s09

Pretty IA, Maupome G (2004). A closer look at diagnosis in clinical dental practice: Part 5. Emerging technologies for caries detection and diagnosis. F Can Dent Assoc, 70(8): 540, 540a-540i.

Ricketts DN, Ekstrand KR, Kidd EA, Larsen T (2002). Relating visual and radiographic ranked scoring systems for occlusal caries detection to histological and microbiological evidence. Oper Dent, 27(3): 231-237.

Schaefer G, Pitchika V, Litzenburger F, Hickel R, Kühnisch J (2018). Evaluation of occlusal caries detection and assessment by visual inspection, digital bitewing radiography and near-infrared light transillumination. Clin Oral Investig, 22(7): 2431-2438. https://doi.org/10.1007/s00784-018-2512-0

Schneiderman A, Elbaum M, Shultz T, Keem S, Greenebaum M, Driller J (1997). Assessment of dental caries with digital imaging fiber-optic transillumination (DIFOTI): In vitro study. Caries Res, 31(2): 103-110. https://doi.org/10.1159/ 000262384

Shoaib L, Deery C, Ricketts DN, Nugent ZJ (2009). Validity and reproducibility of ICDAS II in primary teeth. Caries Res, 43(6): 442-448. https://doi.org/10.1159/ 000258551 
Sidi AD, Naylor MN (1988). A comparison of bitewing radiography and interdental transillumination as adjuncts to the clinical identification of approximal caries in posterior teeth. Br Dent F, 164(1): 15-18. https://doi.org/10.1038/sj.bdj.4806329

Simon JC, Kang H, Staninec M, Jang AT, Chan KH, Darling CL et al. (2017). Near-IR and CP-OCT imaging of suspected occlusal caries lesions. Lasers Surg Med, 49(3): 215224. https://doi.org/10.1002/1sm.22641

Smallridge J (2000). Management of the stained fissure in the first permanent molar. Int $\mathcal{f}$ Paediatr Dent, 10(1): 79-83. https://doi.org/ 10.1046/j.1365-263x.2000.00160.x

Staninec M, Lee C, Darling CL, Fried D (2010). In vivo near-IR imaging of approximal dental decay at 1,310 nm. Lasers Surg Med, 42(4): 292-298. https://doi.org/10.1002/ lsm.20913

Tassery H, Levallois B, Terrer E, Manton DJ, Otsuki M, Koubi S et al. (2013). Use of new minimum intervention dentistry technologies in caries management. Aust Dent F, 58(Suppl 1): 40-59. https://doi. org/10.1111/adj.12049

Tassoker M, Ozcan S, Karabekiroglu S (2020). Occlusal caries detection and diagnosis using visual ICDAS criteria, laser fluorescence measurements, and nearinfrared light transillumination images. Med Princ Pract, 29: 25-31. https://doi.org/ 10.1159/000501257
Wenzel A, Verdonschot EH, Truin GJ, König KG (1992). Accuracy of visual inspection, fiber-optic transillumination, and various radiographic image modalities for the detection of occlusal caries in extracted non-cavitated teeth. F Dent Res, 71(12): 1934-1937. https://doi.org/10 $.1177 / 00220345920710121501$

Yang J, Dutra V (2005). Utility of radiology, laser fluorescence, and transillumination. Dent Clin North Am, 49(4): 739-752. https://doi.org/10.1016/j.cden.2005.05.010

Young DA, Featherstone JD (2005). Digital imaging fiber-optic trans-illumination, F-speed radiographic film and depth of approximal lesions. I Am Dent Assoc, 136(12): 1682-1687. https://doi.org/10 .14219 /jada.archive.2005.0111

Yusof MYPM, Rahman NLA, Asri AAA, Othman NI, Mokhtar IW (2017). Repeat analysis of intraoral digital imaging performed by undergraduate students using a complementary metal oxide semiconductor sensor: An institutional case study. Imaging Sci Dent, 47(4): 233-239. https://doi.org/10.5624/isd.2017.47.4.233 